Copyright $@ 2010$ Institute of Electrical and electronics Engineers, Inc.

All Rights reserved.

Personal use of this material, including one hard copy reproduction, is permitted.

Permission to reprint, republish and/or distribute this material in whole or in part for any other purposes must be obtained from the IEEE.

For information on obtaining permission, send an e-mail message to stds-igr@ieee.org.

By choosing to view this document, you agree to all provisions of the copyright laws protecting it.

Individual documents posted on this site may carry slightly different copyright restrictions.

For specific document information, check the copyright notice at the beginning of each document. 


\title{
Effects of Small Time-Delays on Dynamic Output Feedback Control of Offshore Steel Jacket Structures Subject to Wave-induced Forces
}

\author{
Xian-Ming Zhang, Qing-Long Han* and Dongsheng Han
}

\begin{abstract}
This paper is to investigate the effect of a small time-delay on dynamic output feedback control of an offshore steel jacket structure subject to a nonlinear wave-induced selfexcited hydrodynamic force. Firstly, a conventional dynamic output feedback controller is designed to reduce the internal oscillations of the offshore structure. It is found that the obtained controller is of a large gain in the sense of Euclidean norm, which demands a large control force. Secondly, a small time-delay is introduced intentionally to design a new dynamic output feedback controller such that (i) the controller is of a small gain in the sense of Euclidean norm; and (ii) the internal oscillations of the offshore structure can be dramatically reduced. It is shown through simulation results that purposefully introducing time-delays can be used to improve control performance.
\end{abstract}

\section{INTRODUCTION}

Modern offshore structures are mainly used in oil and gas extraction and are usually built in a water depth of more than 1000 feet. Equipped with a helicopter pad, drilling derrick, cranes, offices and accommodations, generally speaking, these structures are very large, sophisticated and flexible. Since offshore structures are located in a hostile environment, they are typically subject to severe loads due to water currents, waves and wind. In addition, their flexibilities further induce self-excited nonlinear hydrodynamic forces and make themselves large deformations due to nonlinear responses. Therefore, continuing research on the safety of these structures has been conducted in the past decade. One can refer to [4], [3] and references therein.

In order to ensure the safety of an offshore structure, some efforts have been made in the recent years. An easy approach is to increase the stiff of the structure so as to shift the natural frequencies away from the resonant range of frequencies. This requires a large number of construction materials, which leads to a huge cost. Alternatively, passive and active control methods have been proposed to reduce the internal oscillations by regulating the motion of the structure, see, for example, [9], [3]. By placing a tuned mass damper (TMD) onto the top of the structure, both a

This work was supported in part by the Australian Research Council Discovery Project entitled "Variable Structure Control Systems in Networked Environments" (DP0986376) and by Central Queensland University for the Research Advancement Awards Scheme Project "Robust Fault Detection, Filtering, and Control for Uncertain Systems with Time-Varying Delay" (Jan 2006 - Dec 2008) and the Seed Grant Scheme Project entitled " $H_{\infty}$ control and filtering for networked control systems" (Jan 2009 - Dec 2009).

Centre for Intelligent and Networked Systems, and School of Computing Sciences, Central Queensland University, Rockhampton, QLD 4702, Australia

* Corresponding author: Qing-Long Han, Tel. +61 74930 9270; Fax +61 74930 9729; E-mail: q.han@cqu.edu.au

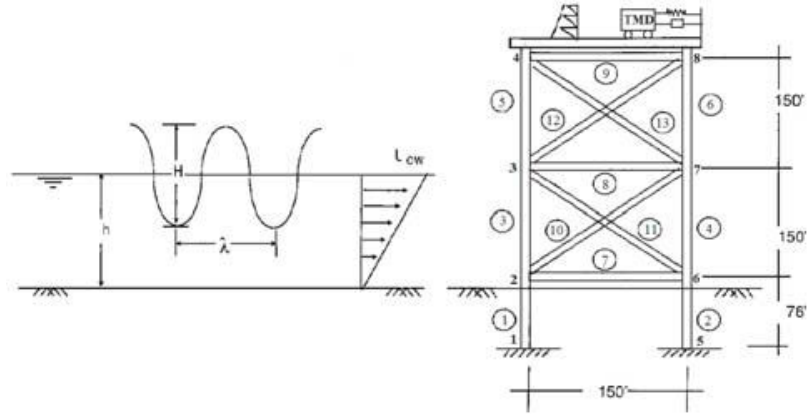

Fig. 1. Steel jacket structure with TMD [14]

passive TMD and an active TMD mechanism to regulate the motion of the structure were discussed in [3]. An active TMD scheme, which aims to design a suitable controller to drive the hydraulic servo, can more effectively reduce the internal oscillations than a passive TMD method. However, for an active TMD mechanism, the controller is usually difficult to be designed mainly due to the self-excited nonlinear hydrodynamic force induced by the flexibility of the structure. For this reason, more attention has been paid to the active TMD control of an offshore structure, and some control methods have been proposed in the recent literature. For instance, in [14], multi-loop feedback control method was introduced: an inner loop is used to stabilize the linear part of the platform dynamical model while an outer loop aims to cope with the nonlinearities to maintain the stability of the whole structure. In [15], two different control schemes, namely nonlinear control and robust linear state feedback control, have been reported. In [11], [10], feedforward and feedback control was studied. The above mentioned methods are implemented by using the system's states. To the best of our knowledge, in the open published literature there is little research on dynamic output feedback control of an offshore structure.

In this paper, we will consider dynamic output feedback control of an offshore steel jacket structure subject to a nonlinear wave-induced force. A conventional dynamic output feedback controller will be first designed by solving a set of LMIs. Then we will investigate whether purposefully in troduced time-delays in dynamic output feedback controllers can be used to improve the control performance.

Throughout this paper, for simplicity, the symmetric term in a symmetric matrix is denoted by *, e.g., $\left[\begin{array}{ll}X & Y \\ * & Z\end{array}\right]=\left[\begin{array}{cc}X & Y \\ Y^{T} & Z\end{array}\right]$

\section{Dynamic Model of an Offshore Structure}

Consider a simple offshore steel jacket platform in Figure 1 [14]. This structure consists of cylindrical steel tube 


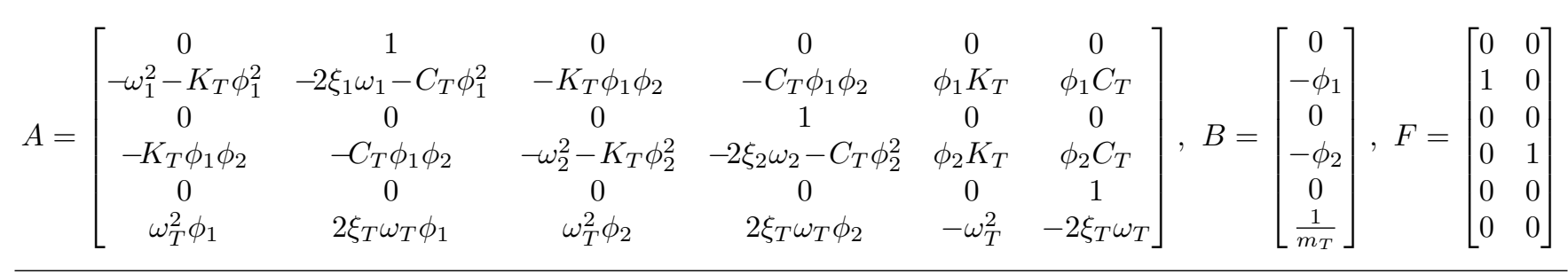

members, and an active TMD mechanism is mounted on the top. One end of the TMD is connected to joint 8 and the other to a hydraulic servo mechanism. The motion of the damper is influenced by the motion of the structure and the hydraulic servo. Since this structure is often exposed to nonlinear hydrodynamic forces, the induced self-excited load by the nonlinear forces usually make the structure internally vibrate. An active TMD control is to design a controller to drive the hydraulic servo so as to reduce the vibration by the motion of the damper. Since the first two modes of vibration are the most dominant, for simplicity, we just take these two modes into account.

The motion equations of the first two modes of vibration with the coupled TMD can be expressed as [15]

$$
\left\{\begin{aligned}
\ddot{z}_{1}= & -2 \xi_{1} \omega_{1} \dot{z}_{1}-\omega_{1}^{2} z_{1}-\phi_{1} K_{T}\left(\phi_{1} z_{1}+\phi_{2} z_{2}\right)+\phi_{1} K_{T} \bar{y} \\
& -\phi_{1} C_{T}\left(\phi_{1} \dot{z}_{1}+\phi_{2} \dot{z}_{2}\right)+\phi_{1} C_{T} \dot{y}-\phi_{1} u+f_{t 1}+f_{t 2}, \\
\ddot{z}_{2}= & -2 \xi_{2} \omega_{2} \dot{z}_{2}-\omega_{2}^{2} z_{2}-\phi_{2} K_{T}\left(\phi_{1} z_{1}+\phi_{2} z_{2}\right)+\phi_{2} K_{T} \bar{y} \\
& -\phi_{2} C_{T}\left(\phi_{1} \dot{z}_{1}+\phi_{2} \dot{z}_{2}\right)+\phi_{2} C_{T} \dot{\bar{y}}-\phi_{2} u+f_{t 3}+f_{t 4} \\
\ddot{\bar{y}}= & -2 \xi_{T} \omega_{T} \dot{\bar{y}}+2 \xi_{T} \omega_{T}\left(\phi_{1} \dot{z}_{1}+\phi_{2} \dot{z}_{2}\right)-\omega_{T}^{2} \bar{y} \\
& +\omega_{T}^{2}\left(\phi_{1} z_{1}+\phi_{2} z_{2}\right)+\frac{1}{m_{T}} u
\end{aligned}\right.
$$

where $z_{1}$ and $z_{2}$ denote the generalized coordinates of vibrational modes 1 and 2 , respectively; $\bar{y}$ is the horizontal displacement of the TMD; $\xi_{1}$ and $\xi_{2}$ are the damping ratio in the first two modes of vibration, respectively; $\omega_{1}$ and $\omega_{2}$ are the natural frequencies of the first two modes of vibration, respectively; $\phi_{1}$ and $\phi_{2}$ are the contributions of first two mode shapes (for the steel jacket platform, $\phi_{1}=-0.003463$ and $\left.\phi_{2}=0.003463\right) ; \xi_{T}$ is the damping ratio of the TMD. We denote the damping, the mass and the stiffness of the TMD by $C_{T}, m_{T}$ and $K_{T}$, respectively; $\omega_{T}=\sqrt{K_{T} / m_{T}}$ is called the natural frequency of the TMD. $u$ is the control action of the system; and $f_{t 1}, f_{t 2}, f_{t 3}, f_{t 4}$ are the nonlinear self-excited hydrodynamic force terms.

Let $x_{1}=z_{1}, x_{2}=\dot{z}_{1}, x_{3}=z_{2}, x_{4}=\dot{z}_{2}, x_{5}=\bar{y}, x_{6}=$ $\dot{\bar{y}}$ and $x:=\left[\begin{array}{llllll}x_{1} & x_{2} & x_{3} & x_{4} & x_{5} & x_{6}\end{array}\right]^{T}$. Rewrite (1) as

$$
\dot{x}(t)=A x(t)+B u(t)+F g(x, t)
$$

where $A, B$ and $F$ are listed on the top of this page; and

$$
g(x, t)=\left[\begin{array}{l}
f_{t 1}+f_{t 2} \\
f_{t 3}+f_{t 4}
\end{array}\right]
$$

As pointed out in [14], the nonlinear function $g(x, t)$ is uniformly bounded and can be assumed to satisfy the following cone-bounding constraint

$$
\|g(x, t)\|_{2} \leq \mu\|x\|_{2}
$$

where $\mu$ is a positive number. In fact, the nonlinear function $g(x, t)$ is usually regarded as a sinusoidal disturbance, one can refer to [12], [13].

In Figure 1, suppose $H=40 \mathrm{ft}, h=250 \mathrm{ft}, \lambda=$ $600 \mathrm{ft}, U_{\text {ow }}=0.4 \mathrm{ft} / \mathrm{sec}$ and the other data of the structure can be referred to [3] or [15]. The natural frequencies of the first two modes of vibration are assumed to be $\omega_{1}=$ 1.818 and $\omega_{2}=10.8717$, respectively. By employing the known data, the coefficient matrices, $A$ and $B$ of (2) can be calculated, which are given in (4), on the top of the next page.

Suppose the wave frequency to be $1.8 \mathrm{rps}$, the nonlinear wave forces can be computed as Appendix A in [15]. When no control is applied to the offshore structure, the responses of the first, second and third floors of the structure subject to nonlinear wave-induced forces are plotted in Figure 2 [15], respectively, from which it is clearly seen that, the uncontrolled responses oscillate with amplitudes of peak to peak about $2.2627 \mathrm{ft}, 2.4518 \mathrm{ft}$ and $2.5379 \mathrm{ft}$, respectively. In what follows, we propose dynamic output feedback schemes to reduce the internal oscillations to guarantee the safety of the structure.

\section{Dynamic Output Feedback Control}

Let $y$ denote the output vector of the system

$$
y(t)=C x(t)
$$

where $C$ is a constant real matrix of appropriate dimensions. The corresponding state space model of the offshore structure can be expressed as

$$
\left\{\begin{array}{l}
\dot{x}(t)=A x(t)+B u(t)+F g(x, t) \\
y(t)=C x(t) \\
x(t)=0, \quad t=0
\end{array}\right.
$$

We seek a dynamic output feedback controller of the form

$$
\left\{\begin{aligned}
\dot{x}_{c}(t) & =A_{K} x_{c}(t)+B_{K} y(t) \\
u(t) & =C_{K} x_{c}(t)+D_{K} y(t)
\end{aligned}\right.
$$

where $x_{c} \in \mathbb{R}^{6}$ and $A_{K}, B_{K}, C_{K}$ and $D_{K}$ are real matrices of appropriate dimensions to be determined, such that the resulting closed loop system is asymptotically stable.

Introduce an augmented vector

$$
\zeta(t):=\left[\begin{array}{ll}
x^{T}(t) & x_{c}^{T}(t)
\end{array}\right]^{T}
$$

then the resulting closed-loop system by (6) and (7) is given by

$$
\dot{\zeta}(t)=\left(A_{0}+H K L\right) \zeta(t)+E^{T} F g(x, t)
$$




$$
A=\left[\begin{array}{cccccc}
0 & 1 & 0 & 0 & 0 & 0 \\
-3.3235 & -0.0212 & 0.0184 & 0.0030 & -5.3449 & -0.8819 \\
0 & 0 & 0 & 1 & 0 & 0 \\
0.0184 & 0.0030 & -118.1385 & -0.1117 & 5.3468 & 0.8822 \\
0 & 0 & 0 & 0 & 0 & 1 \\
-0.0114 & -0.0019 & 0.0114 & 0.0019 & -3.3051 & -0.5454
\end{array}\right], \quad B=\left[\begin{array}{c}
0 \\
0.003445 \\
0 \\
-0.00344628 \\
0 \\
0.00213
\end{array}\right]
$$
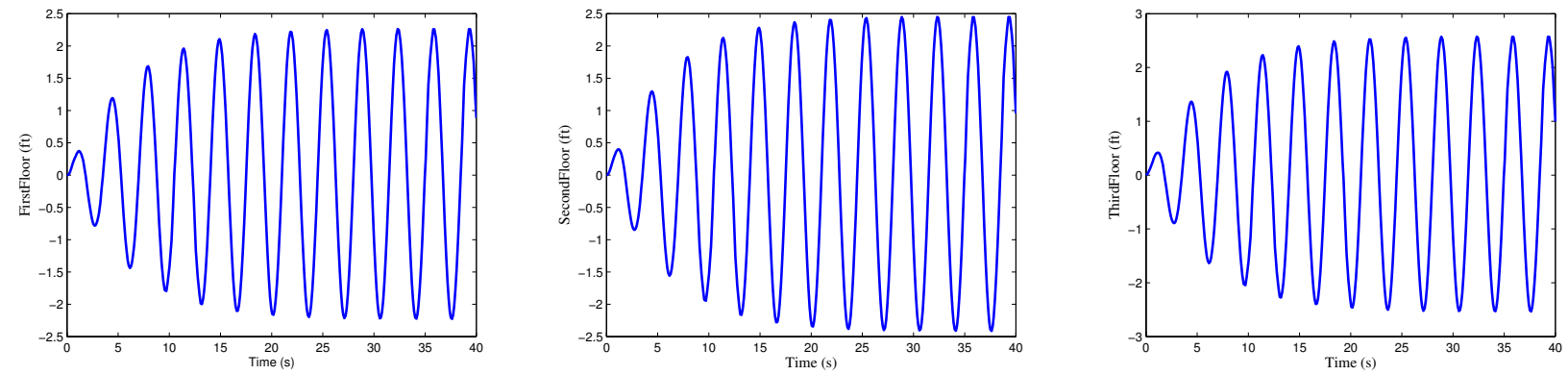

Fig. 2. Responses of the first, second and third floors with no control

where $E=[I 0], A_{0}=\operatorname{diag}\{A, 0\}, H=\operatorname{diag}\{B, I\}, L=$ $\operatorname{diag}\{C, I\}$ and

$$
K:=\left[\begin{array}{ll}
D_{K} & C_{K} \\
B_{K} & A_{K}
\end{array}\right]
$$

For system (8), we have the following result.

Proposition 1: Let $W_{1}$ and $W_{2}$ be the orthogonal complements of $B$ and $C^{T}$, respectively. A dynamic output feedback controller of form (7) is solvable for system (6) if there exist $6 \times 6$ real matrices $X>0, Y>0$ such that

$$
\begin{aligned}
{\left[\begin{array}{cc}
W_{1}^{T}\left(A X+X A^{T}+F F^{T}\right) W_{1} & \mu W_{1}^{T} X \\
* & -I
\end{array}\right]<0 } & <0 \\
{\left[\begin{array}{cc}
W_{2}^{T}\left(Y A+A^{T} Y+\mu^{2} I\right) W_{2} & W_{2}^{T} Y F \\
* & -I
\end{array}\right]<0 } & {\left[\begin{array}{cc}
X & I \\
* & Y
\end{array}\right]>0 }
\end{aligned}
$$

Moreover, if (10)-(12) are feasible on matrix variables $X, Y$, then the controller parameters $K$ defined in (9) can be obtained by the following LMI

$$
\begin{aligned}
P\left(A_{0}+H K L\right) & +\left(A_{0}+H K L\right)^{T} P \\
& +\mu^{2} E^{T} E+P E^{T} F F^{T} E P<0
\end{aligned}
$$

where

$$
P:=\left[\begin{array}{cc}
Y & I \\
N^{T} & 0
\end{array}\right]\left[\begin{array}{cc}
I & X \\
0 & M^{T}
\end{array}\right]^{-1}, \quad M N^{T}=I-X Y
$$

Proof: See the full version of this paper [16].

Now, based on Proposition 1, we design a dynamic output feedback controller for system (2) with (4). Let

$$
C=\left[\begin{array}{llllll}
1 & 0 & 0 & 0 & 0 & 0 \\
0 & 0 & 1 & 0 & 0 & 0 \\
0 & 0 & 0 & 0 & 1 & 0
\end{array}\right]
$$

Applying Proposition 1 , for $\mu=1$, the obtained dynamic output feedback controller, which is denoted by $K_{1}$, is given in (7) with $K_{1}:=\left[\begin{array}{c|c}A_{K} & B_{K} \\ \hline C_{K} & D_{K}\end{array}\right]$ being shown on the top of next page. Clearly, the controller $K_{1}$ is of a large gain in the sense of Euclidean norm, namely, $\left\|K_{1}\right\|_{2}=4.1308 \times 10^{8}$, which demands a large control force. It is worth pointing out that this feature remains even for small value of $\mu$. For example, taking $\mu=0.01$ and applying proposition 1 yields a dynamic output feedback controller $K_{2}$, which is also of a large gain with $\left\|K_{2}\right\|_{2}=5.6472 \times 10^{8}$. Figures 3 depicts the responses of the first, second, third floors of the offshore structure under the control of $K_{2}$. From these figures, we can find that the oscillation magnitudes of peak to peak have been reduced from $2.2627 \mathrm{ft}, 2.4518 \mathrm{ft}, 2.5379 \mathrm{ft}$ to $0.5433 \mathrm{ft}, 0.5967 \mathrm{ft}$ and $0.6388 \mathrm{ft}$, respectively.

\section{Dynamic Output Feedback Control By INTRODUCING DELAYED MEASUREMENTS}

In practical feedback control systems, small time-delays in the control action are inevitable because of involved dynamics of actuators and sensors. As is seen from the previous section, the obtained dynamic output feedback controller is of a large gain in the sense of Euclidean norm. In this section, we are interested in investigating the effect of a small timedelay on dynamic output feedback control of the offshore structure. More specifically, we introduce a small time-delay $h>0$ when we measure the output of the system, i.e.

$$
y(t)=C x(t-h)
$$

The corresponding state space model of the offshore structure is stated as

$$
\left\{\begin{array}{l}
\dot{x}(t)=A x(t)+B u(t)+F g(x, t) \\
y(t)=C x(t-h) \\
x(t)=0, \quad t \in[-h, 0] .
\end{array}\right.
$$

We now design a dynamic output feedback controller of form (7) based on the system (17). The resulting closed-loop 


$$
K_{1}=10^{8} \times\left[\begin{array}{rrrrrr|rrr}
-0.0058 & -0.0083 & 0.0023 & -0.0011 & -0.0064 & -0.0000 & -0.0035 & -0.0015 & -0.0107 \\
-0.2918 & -0.4229 & 0.1174 & -0.0554 & -0.3246 & -0.0007 & -0.1780 & -0.0769 & -0.5410 \\
1.2906 & 1.2299 & -0.2677 & 0.1075 & 0.9412 & 0.0028 & 0.4031 & -0.5884 & 1.5677 \\
1.4708 & -0.5941 & 0.4779 & -0.3062 & -0.4671 & 0.0020 & -0.7372 & -3.5625 & -0.7830 \\
-1.1264 & -1.3226 & 0.4061 & -0.1785 & -1.2684 & -0.0012 & -0.1052 & 0.2773 & -2.1279 \\
-1.3491 & -1.2400 & -0.2681 & 0.1182 & 0.8629 & -0.0126 & -3.2107 & -0.2128 & 1.5399 \\
\hline-2.9911 & 1.3116 & 0.0897 & -0.0887 & 0.2414 & 0.0026 & 1.0962 & -1.7721 & 0.3633
\end{array}\right]
$$
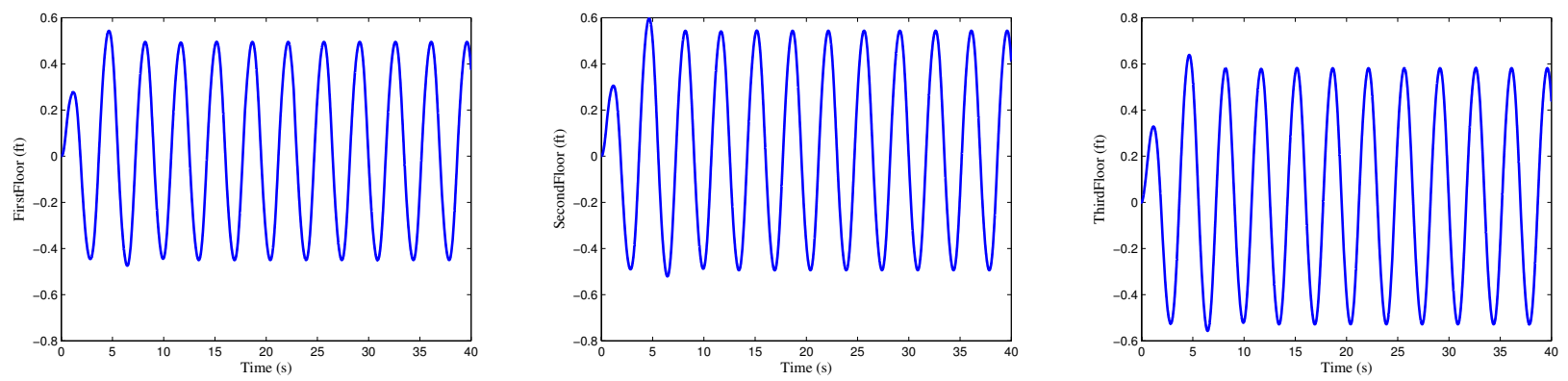

Fig. 3. Responses of the first, second and third floor under the control of $K_{2}$

system by (17) and (7) is

$$
\dot{\zeta}(t)=\left(A_{0}+H K L_{1}\right) \zeta(t)+H K L_{2} E \zeta(t-h)+E^{T} F g(x, t)
$$

where $A_{0}, H, K$ are the same as those in (8) and

$$
L_{1}:=\left[\begin{array}{cc}
0 & 0 \\
0 & I
\end{array}\right], \quad L_{2}:=\left[\begin{array}{c}
C \\
0
\end{array}\right]
$$

We now state and establish the following stability criterion.

Proposition 2: For given positive scalars $\mu$ and $h$, the system (18) is asymptotically stable if there exist real matrices $P>0, Q>0$ and $R>0$ of appropriate dimensions such that matrix inequality (20), on the top of next page, holds.

Proof: See the full version of this paper [16].

Proposition 2 provides a delay-dependent stability criterion for the nonlinear system (18). However, this condition cannot be used to design the controller parameters directly due to some nonlinear terms, such as $P H K L_{1}$ and $P H K L_{2}$ etc. in matrix inequality (20). In what follows, we propose a controller design method based on Proposition 2. Similar to the proof of Proposition 1, a sufficient condition of the existence of a desired controller can be obtained, which is stated in the following proposition.

Proposition 3: Let $W_{1}$ and $\left[\begin{array}{ll}W_{2}^{T} & W_{3}^{T}\end{array}\right]^{T}$ be the orthogonal complements of $C^{T}$ and $\left[\begin{array}{ll}B^{T} & B^{T}\end{array}\right]^{T}$, respectively. Matrix inequality (20) is feasible on matrix variable $K$ if and only if there exist real $6 \times 6$ matrices $X>0, Y>0, Q>0$ and $R>0$ such that (12) and

$$
\begin{gathered}
{\left[\begin{array}{cccc}
\Xi_{11} & R W_{1} & Y F & h A^{T} R \\
* & W_{1}^{T}(-Q-R) W_{1} & 0 & 0 \\
* & * & -I & h F^{T} R \\
* & * & * & -R
\end{array}\right]<0} \\
\multicolumn{2}{c}{\Omega:=\left[\begin{array}{cc}
\Omega_{11} & W_{2}^{T} X R \\
* & -Q-R
\end{array}\right]<0}
\end{gathered}
$$

where

$$
\begin{aligned}
\Omega_{11}:= & W_{2}^{T}\left[A X+X A^{T}+X\left(Q-R+\mu^{2} I\right) X\right] W_{2} \\
& +\left(W_{2}+W_{3}\right)^{T} F F^{T}\left(W_{2}+W_{3}\right)+W_{2}^{T} X A^{T} W_{3} \\
& +W_{3}^{T} A X W_{2}-h^{-2} W_{3}^{T} R^{-1} W_{3} \\
\Xi_{11}:= & Y A+A^{T} Y+Q-R+\mu^{2} I .
\end{aligned}
$$

One can see that matrix inequality (22) is still nonlinear on matrix variables, which is a non-convex feasible problem. Now, we will convert this non-convex feasible problem into a nonlinear minimization problem subject to a set of LMIs. Define $J:=\operatorname{diag}\{I, X\}$, then pre- and post-multiplying both sides of $\Omega$ in (22) by $J^{T}$ and its transpose, respectively, yield

$$
J^{T} \Omega J=\left[\begin{array}{cc}
\Omega_{11} & W_{2}^{T} X R X \\
* & -X Q X-X R X
\end{array}\right]<0
$$

Introducing two new matrix variables $S>0$ and $Z>0$ such that

$$
X R X \geq S, \quad X Q X \geq Z
$$

which are equivalent to, respectively

$$
\left[\begin{array}{cc}
R & X^{-1} \\
X^{-1} & S^{-1}
\end{array}\right] \geq 0, \quad\left[\begin{array}{cc}
Q & X^{-1} \\
X^{-1} & Z^{-1}
\end{array}\right] \geq 0
$$

Notice that

$$
\left[\begin{array}{cc}
-W_{2}^{T} X R X W_{2} & W_{2}^{T} X R X \\
* & -X R X
\end{array}\right] \leq\left[\begin{array}{cc}
-W_{2}^{T} S W_{2} & W_{2}^{T} S \\
* & -S
\end{array}\right]
$$

One can see that matrix inequality (23) is implied by the following

$$
\left[\begin{array}{cc}
\tilde{\Omega}_{11} & W_{2}^{T} S \\
* & -Z-S
\end{array}\right]<0
$$

where

$$
\tilde{\Omega}_{11}:=W_{2}^{T}\left[A X+X A^{T}+X\left(Q+\mu^{2} I\right) X-S\right] W_{2}
$$




$$
\begin{aligned}
\Theta & :=\left[\begin{array}{cccc}
\Theta_{11} & P H K L_{2}+E^{T} R & P E^{T} F & h\left(A_{0}+H K L_{1}\right)^{T} E^{T} R \\
* & -Q-R & 0 & h H K L_{2}^{T} E^{T} R \\
* & * & -I & h F^{T} R \\
* & * & * & -R
\end{array}\right]<0 \\
\Theta_{11} & :=P\left(A_{0}+H K L_{1}\right)+\left(A_{0}+H K L_{1}\right)^{T} P+E^{T}\left(Q-R+\mu^{2} I\right) E .
\end{aligned}
$$

$$
\begin{aligned}
& +\left(W_{2}+W_{3}\right)^{T} F F^{T}\left(W_{2}+W_{3}\right)+W_{2}^{T} X A^{T} W_{3} \\
& +W_{3}^{T} A X W_{2}-h^{-2} W_{3}^{T} R^{-1} W_{3}
\end{aligned}
$$

By using Schur complement, (24) is equivalent to

$$
\left[\begin{array}{cccc}
\breve{\Omega}_{11} & W_{2}^{T} S & W_{2}^{T} X & \mu W_{2}^{T} X \\
* & -Z-S & 0 & 0 \\
* & * & -Q^{-1} & 0 \\
* & * & * & -I
\end{array}\right]<0
$$

where

$$
\begin{aligned}
\breve{\Omega}_{11}:= & W_{2}^{T}\left[A X+X A^{T}-S\right] W_{2}-h^{-2} W_{3}^{T} R^{-1} W_{3} \\
& +\left(W_{2}+W_{3}\right)^{T} F F^{T}\left(W_{2}+W_{3}\right) \\
& +W_{2}^{T} X A^{T} W_{3}+W_{3}^{T} A X W_{2}
\end{aligned}
$$

Therefore, setting $\bar{R}=R^{-1}, \bar{X}=X^{-1}, \bar{S}=S^{-1}, \bar{Z}=$ $Z^{-1}, \bar{Q}=Q^{-1}$, and similar to the proof of Proposition 2, we can derive a new sufficient condition for the existence of the dynamic output feedback controller, which is formulated in the following result.

Proposition 4: Let $W_{1}$ and $\left[\begin{array}{ll}W_{2}^{T} & W_{3}^{T}\end{array}\right]^{T}$ be the orthogonal complements of $C^{T}$ and $\left[\begin{array}{ll}B^{T} & B^{T}\end{array}\right]^{T}$, respectively. For given scalars $\mu>0$ and $h>0$, the dynamic output feedback control problem for system (17) is solvable if there exist $6 \times 6$ real matrices $X>0, Y>0, Q>0, R>0, \bar{R}>$ $0, \bar{X}>0, S>0, \bar{S}>0, Z>0, \bar{Z}>0$ and $\bar{Q}>0$ such that (12), (21) and

$$
\begin{gathered}
{\left[\begin{array}{ccccc}
\Upsilon & W_{2}^{T} S & \left(W_{2}^{T}+W_{3}^{T}\right) F & W_{2}^{T} X & \mu W_{2}^{T} X \\
* & -Z-S & 0 & 0 & 0 \\
* & * & -I & 0 & 0 \\
* & * & * & -\bar{Q} & 0 \\
* & * & * & * & -I
\end{array}\right]<0} \\
\quad\left[\begin{array}{cc}
R & \bar{X} \\
\bar{X} & \bar{S}
\end{array}\right] \geq 0, \quad\left[\begin{array}{cc}
Q & \bar{X} \\
\bar{X} & \bar{Z}
\end{array}\right] \geq 0 \\
\quad R \bar{R}=I, X \bar{X}=I, S \bar{S}=I, Z \bar{Z}=I, Q \bar{Q}=I
\end{gathered}
$$

where

$$
\begin{aligned}
\Upsilon:= & W_{2}^{T}\left[A X+X A^{T}-S\right] W_{2}+W_{2}^{T} X A^{T} W_{3} \\
& +W_{3}^{T} A X W_{2}-h^{-2} W_{3}^{T} \bar{R} W_{3} .
\end{aligned}
$$

Proposition 4 is based on a set of LMIs subject to equality constraints. By employing the cone complementary method proposed in [5], it can be converted into a nonlinear minimization problem (NMP) subject to LMIs, which is stated in the following.

\section{Nonlinear Minimization Problem (NMP)}

Minimize $\operatorname{Tr}(\bar{X} X+\bar{R} R+\bar{Q} Q+\bar{Z} Z+\bar{S} S)$
Subject to (12), (21), (26), (27) and

$$
\begin{aligned}
& {\left[\begin{array}{cc}
R & I \\
I & \bar{R}
\end{array}\right] \geq 0,\left[\begin{array}{cc}
X & I \\
I & \bar{X}
\end{array}\right] \geq 0,\left[\begin{array}{cc}
Q & I \\
I & \bar{Q}
\end{array}\right] \geq 0,} \\
& {\left[\begin{array}{cc}
Z & I \\
I & \bar{Z}
\end{array}\right] \geq 0,\left[\begin{array}{cc}
S & I \\
I & \bar{S}
\end{array}\right] \geq 0 .}
\end{aligned}
$$

The following iterative algorithm can be used to solve the above NMP.

Algorithm 1 Solve the NMP (29).

Step 1 Find a feasible set $\left(X^{0}, Y^{0}, Q^{0}, R^{0}, S^{0}, Z^{0}, \bar{X}^{0}, \bar{Q}^{0}, \bar{R}^{0}, \bar{S}^{0}, \bar{Z}^{0}\right)$ satisfying 12), (21), (26), (27) and (30), (31). Set $l=0$.

Step 2 Solve the following LMI problem for the matrix variables $(X, Y, Q, R, S, Z, \bar{X}, \bar{Q}, \bar{R}, \bar{S}, \bar{Z})$ :

Minimize $\operatorname{Tr}\left(\begin{array}{l}\bar{X}^{l} X+X^{l} \bar{X}+\bar{R}^{l} R+R^{l} \bar{R}+\bar{Q}^{l} Q \\ +Q^{l} \bar{Q}+\bar{Z}^{l} Z+Z^{l} \bar{Z}+\bar{S}^{l} S+S^{l} \bar{S}\end{array}\right)$

Subject to $(12),(21),(26),(27)$ and $(30),(31)$

$$
\text { Set } \begin{aligned}
X^{l+1} & =X, Q^{l+1}=Q, R^{l+1}=R, Z^{l+1}=Z, \\
S^{l+1} & =S, \bar{X}^{l+1}=\bar{X}, \bar{Q}^{l+1}=\bar{Q}, \bar{R}^{l+1}=\bar{R}, \\
\bar{Z}^{l+1} & =\bar{Z}, \bar{S}^{l+1}=\bar{S} .
\end{aligned}
$$

Step 3 If matrix inequality (22) and

$$
\left|\operatorname{Tr}\left(\begin{array}{c}
\bar{X}^{l} X+X^{l} \bar{X}+\bar{R}^{l} R+R^{l} \bar{R}+\bar{Q}^{l} Q \\
+Q^{l} \bar{Q}+\bar{Z}^{l} Z+Z^{l} \bar{Z}+\bar{S}^{l} S+S^{l} \bar{S}
\end{array}\right)-10 n\right|_{(32)}
$$

where $\varepsilon$ is a prescribed sufficiently small positive number, are satisfied, then set $l=l+1$ and go to Step 2. If one of the conditions (22) and (32) is not satisfied within a specified number of iterations, then exit.

Finally, if the nonlinear minimization problem (29) is feasible on the matrix variables $R, Q$ etc., then the desired dynamic output feedback controller of form (7) can be obtained by solving the LMI (20) on the matrix variable $K$ in (9) with the known $R, Q$ and $P$ of form (14).

Now, we are in position to design a dynamic output feedback controller for system (2) with (4) based on Proposition 3 incorporating with Algorithm 1. Setting $h=0.02$, for $\mu=1$, the dynamic output feedback controller, which is denoted by $K_{3}$, can be derived, and the controller parameters $K_{3}:=\left[\begin{array}{c|c}A_{K} & B_{K} \\ \hline C_{K} & D_{K}\end{array}\right]$ are given on the top of this page.

It is worth noting that the controller gain of $K_{3}$ with $\left\|K_{3}\right\|_{2}=6.6224 \times 10^{4}$ is much smaller than those of $K_{1}$ with $\left\|K_{1}\right\|_{2}=4.1308 \times 10^{8}$ and $K_{2}$ with $\left\|K_{1}\right\|_{2}=$ 


$K_{3}=\left[\begin{array}{rrrrrr|rrr}-19.821 & 1.3551 & 4.6275 & -5.0287 & -0.0824 & -0.3689 & 0.0042 & -0.0028 & 0.0008 \\ -1.9217 & -1.3374 & -1.2510 & 1.3649 & 0.0221 & 0.0997 & 0.0103 & 0.0013 & 0.0024 \\ 44.687 & -2.4412 & -5.3613 & 2.3260 & -0.5188 & -2.5452 & 0.6109 & -118.7021 & -3.5789 \\ 53.866 & -1.1599 & -2.9063 & -3.0366 & -0.5630 & -2.9301 & 1.2531 & -119.9537 & -3.4823 \\ 52.437 & 9.1799 & 4.4460 & 1.8615 & -0.7438 & -2.9704 & 19.6555 & -80.3220 & 2.1280 \\ 574.38 & 129.27 & 116.50 & -97.968 & -0.4820 & -38.056 & 234.6118 & -1331.8 & 10.8054 \\ \hline-62745 & 2519 & 14215 & -15450 & -253 & -1133 & -0.0016 & 0.3327 & 0.0059\end{array}\right]$
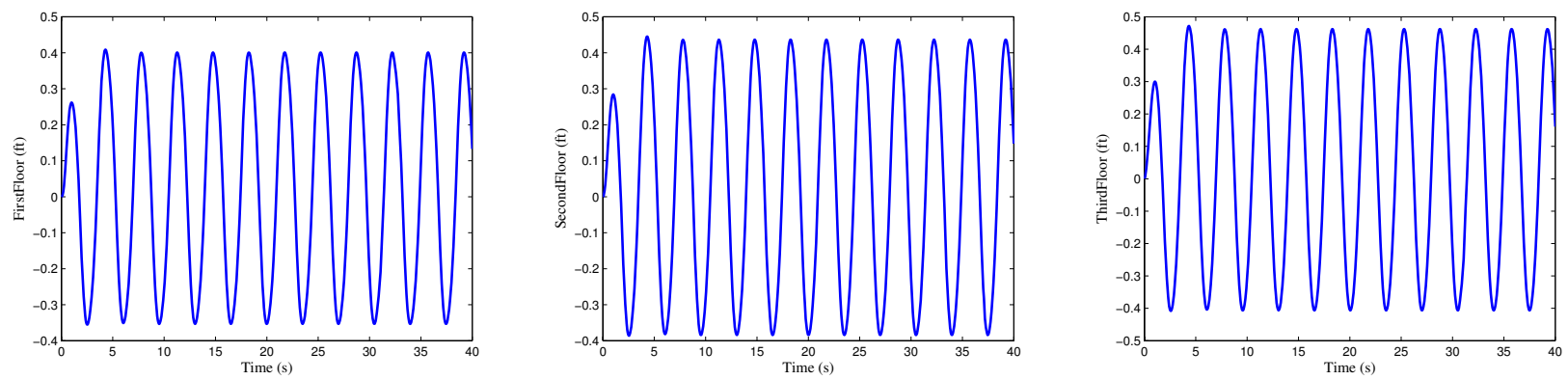

Fig. 4. Responses of the first, second and third floors under the control of $K_{3}$

$5.6472 \times 10^{8}$. So it is more easily implemented than $K_{1}$ and $K_{2}$ for the offshore structure. In addition, we plot the response curves of the first, second and third floors of the offshore structure via the controller of $K_{3}$, which are shown in Figure 4. Clearly, the oscillation amplitudes of peak to peak are apparently reduced from the uncontrolled values of $2.2627 \mathrm{ft}, 2.4518 \mathrm{ft}, 2.5739 \mathrm{ft}$ to $0.4088 \mathrm{ft}, 0.4451 \mathrm{ft}, 0.4716 \mathrm{ft}$, respectively. Therefore, by appropriately introducing a small time-delay into the output channel, the designed controller is of a small gain in the sense of Euclidean norm, which demands a small control force, and the internal oscillation of the offshore structure can be dramatically reduced.

Remark 1: For an offshore steel jacket structure, the system parameters are usually subject to uncertainty. In this case, the proposed method can easily be extended to design a robust dynamic output feedback controller by intentionally introducing a small time-delay to make the uncertain offshore steel jacket structure work in a safe environment. Due to page limitation, it is omitted.

\section{CONCLUSions}

We have investigated the effect of a small time-delay on dynamic output feedback control of an offshore structure subject to a nonlinear wave-induced force. We have found that appropriately introducing a small time-delay into the output channel, the controller is of a small gain in the sense of Euclidean norm, which demands a small control force, and the internal oscillations of the offshore structure can be dramatically reduced. Simulation results have confirmed our finding.

\section{REFERENCES}

[1] S. Boyd, L. EL Ghaoui, E. Feron and V. Balakrishnam, Linear matrix inequalities in system and control, SIAM. Philadelphia, 1994.
[2] M. Abdel-Rohman, Control of nonlinear vibrations in civil structures, Kuwait University Press, 1993.

[3] M. Abdel-Rohman, "Structure control of a steel jacket platform", Structural and Engineering Mechanics, vol. 4, 1996, pp. 125-138.

[4] S. Chakrabati, Hydrodynamics of offshore structures, Springer, Berlin, 1987.

[5] L. El Ghaoui, F. Oustry and M. Ait Rami, "A cone complementarity linearization algorithms for static output feedback and related problems", IEEE Transactions on Automatic Control, vol. 42, 1997, pp. 1171-1176.

[6] J.K. Hale and S.M. Verduyn Lunel, Introduction to Functional Differential Equations, New York: Springer-Verlag, 1993.

[7] Q.-L. Han, "Absolute stability of time-delay systems with sectorbounded nonlinearity", Automatica, vol. 41, 2005, pp. 2171-2176.

[8] E. Jeung, J. Kim and H. Park, " $H_{\infty}$ output feedback controller design for linear systems with time-varying delayed state", IEEE Transactions on Automatic Control, vol. 43, 1998, pp. 971-974.

[9] K. Kawano, "Active control effects on dynamic response of offshore structure", In Proceedings of the 3rd ISOPE, Singapore, 1993, pp. 594-598.

[10] H. Ma, G.Y. Tang and Y.D. Zhao, "Feedforward and feedback optimal for offshore structures subject to irregular wave forces", Ocean Engineering, vol. 33, 2006, pp. 1105-1117.

[11] J. Suhardjo and A. Kareem, "Feedback-feedforward control of offshore platforms under random waves", Earthquake Engineering and Structure Dynamics, vol. 30, 2001, pp. 213-235.

[12] G.Y. Tang, S.S. Lu and R. Dong, "Optimal sliding mode control for linear time-delay systems with sinusoidal disturbances", Journal of Sound and Vibration, vol. 304, 2007, pp. 263-271.

[13] G.Y. Tang, B.L. Zhang, Y.D. Zhao and S.M. Zhang, "Optimal Sinusoidal Disturbances Damping for Singularly Perturbed Systems with Time-delay", Journal of Sound and Vibration, vol. 300, 2007, pp. 368378.

[14] M.J. Terro, M.S. Mahmoud and M. Abdel-Rohman, "Multi-loop feedback control of offshore steel jacket platforms", Computers and Structures, vol. 70, 1999, pp. 185-202.

[15] M. Zribi, N. Almutairi, M. Abdel-Rohman and M. Terro, "Nonlinear and robust control schemes for offshore steel jacket platforms", Nonlinear Dynamics, vol. 35, 2004, pp. 61-80.

[16] X.-M. Zhang, Q.-L. Han and D.-S. Han, "Effects of small time-delays on dynamic output feedback control of offshore steel jacket structures subject to wave-induced forces", Full version of this paper. 